\title{
Women Brain Foods: Increased Basal Antioxidant Capacity Effects on Mental Cognition and Carcinogenic Diseases
}

\author{
PiMann Getsemi* \\ (ORCiD: 0000-0003-0579-8966) \\ The White House, 1600 Pennsylvania Ave \\ NW, Washington, DC 20500 \\ International Federation of Red Cross and \\ Red Crescent Societies, Geneva 19 Switzerland
}

May 26, 2018

Cognitive improvement efforts evaluated by eating foods rich in antioxidants, and vitamins for first-year college students; significant increments in eating fast foods, certain vitamins, high amounts of several antioxidants or stimulants consumed just before stressful exams, long term overdose on such drugs, breathing toxic air pollutants, would cause illness and fatal adverse effects on scientific societies.

In 1990s, antioxidants came to public attention when researchers began finding a solution for treatment of aging, and cancer. Key research models developed to understand free radical damage, caused by oxygen-based reactions, contribute to chronic diseases. Deficit of antioxidants and vitamins in the common diet should lead to "cognitiva indigestionem" (cognitive impairment), dementia, and Alzheimer disease.[10] Definition of 'cognition' word dates back to the 15 th century, but it traditionally comes from an earlier era when philosophers' efforts leaded to a true reform in logic.[2] Around the last century, a few years after Piaget's cognitive development published, Miller (1960) recognized that the human mind can be understood using an information-processing model.[8][5][7] We can consider high-order thinking depends upon our ability to create mental representations. Stimulant drugs, such as amphetamines and methamphetamines, which are made for treatment of attention deficit hyperactivity disorder have a long history. These drugs had

*Email address: peiman.ghasemi@aol.com — The White House and the IFRC wont be responsible to answer any questions. To send your comments, only use the (personal) email address above, please. 
been used since over a hundred years ago, and during the World War, and still they are being used to treat some medical conditions, but also they are highly addictive, with a history of abuse. These drugs could help us by improving our attention, concentration, and mental representations and cognition. A healthy diet should contain all the essential nutrition such as vitamins, minerals, trace elements, essential amino acids and fatty acids, polyphenols, fluid, fiber, and adequate calories. Earlier, European civilizations - communities of the 16-18th centuries - not only knew how to eat, but what to eat to keep being healthy, and they began eating caviar and avocados, and then - in early $18 \mathrm{~s}$ - a doctor, as one of the first dietitians, began publicly recommending his diet for everyone suffering from obesity. Some experts believe, modern humans eating habits is derived from ancient stone-age. Asian civilizations and Mediterranean peoples each has been adapted to special, selective, eating habits, which currently in the 21st century yet is remained as healthiest balanced diets around the world, containing a variety of such amino-acids, carbs, and other nutrients.[6] Moreover, some forms of smart pills were used since the World War II. So smart pills were given to air force pilots, and some other volunteers, by America, Japan, Great Britain, and Germany to improve their average mental functions during major missions. But, those days, there was a very basic understanding of foods and fruits preventing the aging process either at cellular level or directly by protecting body from free radicals' damage. There was no understanding of modern artificial nootropics and cognitive enhancement drugs, before early 1960s. Little understanding of brain and neuroscience was gained by those people. ${ }^{1}$ Since late 1960s, following publication of "Cognitive Psychology", hard efforts developed towards making smart-drugs for enhancement of mental cognition by scientists and big pharmaceutical companies. The first cognitive enhancer drug (nootropic drug), the earliest Racetam, created in 1964. Earlier drugs were just including stimulant drugs (for treatment of ADHD) until scientists made the first Racetam.

\footnotetext{
${ }^{1} \mathrm{Mr}$. Santiago Ramón y Cajal is considered a father of neuroscience in modern period.
} 


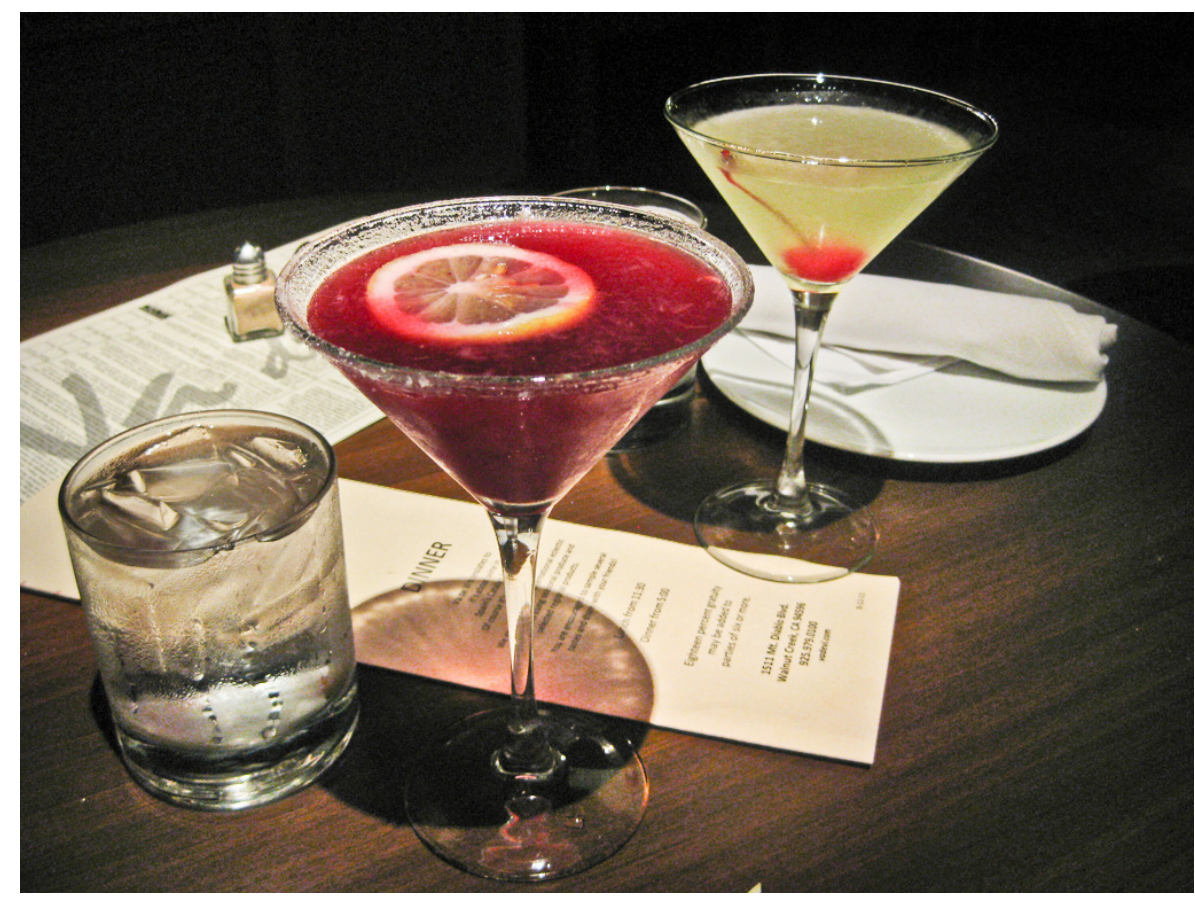

Cocktail (blueberry, lemon drop \& Appletini)

Most of the girls, our youth and eager college students, enjoy healthy morning light meals, i.e. eating yogurt parfait, a light homemade sandwich, or pack an extra snack and bring to the school or college.[9] Eating simple foods is better than going to a burger shop, eating a big burger contains lots of fats. Berries are rich sources of vitamins and antioxidants. Mental antioxidants, and our efforts for decrement of the aging process makes inevitable ties to another important issue, to be discussed here, the subject of carcinogenesis and publics immunization. Antioxidants were not discovered for the first time in late 20th century. In 1929, Hopkins receives the Nobel Prize for discovering vitamins since he demonstrated that unknown accessory factors found in milk, other than carbohydrates, proteins, and fats were necessary for growth in rats.[1] Identification of vitamin $\mathrm{A}, \mathrm{C}$, and $\mathrm{E}$ as antioxidants revolutionized the fields of biochemistry and cellular biology. Antioxidants fight free radicals. Free radicals are nasty chemicals, capable of damaging cells and genetic materials. Free radicals are inevitable byproducts of turning food into energy. The remaining percentage are found in the food you eat and the air you breathe. An antioxidant is a molecule that inhibits the oxidation of other molecules by stabilizing free radicals that can cause cell damage by attaching to other molecules and prompting cells to grow abnormally or by interfering with normal cell functions, including those in the brain. Diets rich in fruits and vegetables are considered to be an excellent and balanced source of antioxidants. Some minerals and vitamins also have a role as antioxidants; 
these include vitamin $\mathrm{C}$, vitamin $\mathrm{E}$ and its isomers (tocopherols and tocotrienols), and selenium. Vitamin A, C, and E act as organic compounds, essential nutrients, and potent antioxidants. However, recent studies relating to the last several years, showed unpredictable results. Some recent studies showed increased rate of mortality over increased consumption of beta carotene, vitamin $\mathrm{E}$, higher doses of vitamin $\mathrm{A}$, and also for $\mathrm{N}$-acetyl cysteine (NAC) a potent antioxidant, as dietary supplements. Research is showing promise for the ability of coenzyme Q10 to affect symptoms related to neurological disease. Since coQ10 functions in the mitochondria, diseases characterized by dysfunction of the mitochondria due to the aging process are improved by coQ10 supplementation. CoQ10 assists with the production of energy and as an antioxidant in cell membranes. A healthy diet plan should be arranged precisely if you want it as a truly good answer to the needs of female college students. High quality foods that contain a balanced quantity of natural vitamins, minerals, and antioxidants are required for a healthy diet. Consumption of fast foods, fats, sweets (such as candies, cookies, and syrups), soda, processed meat, and even a high amount of carton fruit juice and a high amount of milk consumption are getting considered as one of the great causes of inflammation, depression, diabetes and cancer. These foods shrink your brain. A variety of colorful fruits needed for your body cells, organs, and the brain to work well. Fish is a vital food for improvement of mental cognition, and for your brain to work healthy. Without consuming cod fish, salmon, mackerel, occasionally a moderate amount of fish roe (don't get confused with liquid constant in physics), and shrimps it's impossible for your brain to work well. Women are the hopes of the planet towards flourishing American communities and fighting violation. And they must secure their scientific innovational foundations in the United States. Surely Einstein (in early years of his life) enjoyed serving fish and seafood, but mostly he had his chicken eggs and mushrooms on the table for breakfast. But it is possible that Sir Isaac Newton had consumed more fish and some fish eggs. But what about women. Marie Curie, a giant of science, liked to eat tea with bread and butter. She worked too much with radioactive materials and $\mathrm{X}$ rays as much as it probably caused her sickness and death. It is believed King Lemuel of Massa noticeably mentioned tea as the strong drink, along the other books of the Bible. As you have learnt, when you eat some food, the body cells use oxygen to break it down and burn it as a fuel for energy, the process makes free radicals that can cause cancer, or damage the body cells through several ways. During the process, oxidative stress is another important harmful factor. Stress produces peroxides and free radicals and it would damage all components of the cell, including proteins, lipids, and DNA. Cancer cells divide fast and rapidly. They no longer answer to many signals that control cellular growth and death. To date, the public scientific societies have an understanding that a higher level of antioxidants leads to a lower rate of being seek by cancer. However there is an issue we must consider that if you have high cancer family background or tumor or you go under radiation/chemo therapy for killing cancer cells you would gain a negative result following above a minimum level consumption of 
antioxidants, - moreover, patients and those ill individuals may receive dangerous drugs to increase oxidative stress and targeting cancer cells by ROS-mediated mechanisms - A very high level consumption of natural antioxidants to improve mental cognitive functions, for patients with cancer background is considered as another risk factor too; as it would increase tumors and carcinogenic cells in your body for data analysis strongly suggest that an increase in basal antioxidant capacity can contribute to cancer development.[11] East Asian people love drinking green tea, eating okra, avocado, and asparagus with meal. Another fancy is Karaoke in East Asian countries, which for an instance, is tried by boys at Japanese festivals each year. These fruits and amusing activities of Asian people reduce their stress level and the body aging process. Reduce your stress level as much as possible. When I was a child it made me so happy to watch diver movies in underwater world. Let's mention and define another minor but important issue now. Since, recent studies show that above average consumption of antioxidants in stressful environments, potentially would cause cancers. However, eating cherry tomatoes, almonds, berries, green leafy vegetables, ginger roots, and drinking kefir seriously protect your family to get seek because oxidative stress. Also, however if you are healthy and don't taking anti-inflammatory drugs, but you feel stressed in last few weeks of each college semester near your examination dates your doctor must prescribe you an antianxiety drug, such as a benzodiazepine drug. Alprazolam belongs to benzodiazepines class drugs. It works by enhancing the effects of Gamma-Aminobutyric acid (GABA). Benzodiazepines are abusive, and following a long-term usage may cause mental problems. A modern drug, Noopept, an effective nootropic, and anti-inflammatory drug, in some forms even may prevent you getting cancer.

A small plate of blueberries (Vaccinium corymbosum) consumed with some oatmeal mixed with a very small cup of low-fat milk is including beneficial antioxidants which protects your body and your brain to get aged. Blueberries are vitamin-rich berries that boost brain health via their high content of antioxidants, specifically flavonoids. And daily consumption has been found to slow memory impairments and motor coordination that are typically associated with aging. Health benefits, from preventing or treating dementia to improving memory, would be sensible after a few weeks of consumption. Furthermore, during hot days you must make and serve fresh smoothies, both highly premium probiotic smoothies and probiotic yoghurts are an acceptable and delicious habitual alternative - The term probiotic is currently used to name ingested microorganisms associated with benefits for humans and animals. Probiotics are live bacteria and yeasts that are good for your health, especially your digestive system. There is a vast variety of bacteria, living in our body. It's including the both good and bad bacteria. Probiotics are often called good bacteria - For sure enough fresh mineral water is vital for body to function well too. You may serve eggs, traditional premium quality chesses especially for cooked breakfasts (including artisanal chesses, some mozzarella and some cheddar, etc.), tomatoes, potatoes, beans, green beans and pies, and 
mushrooms in the morning with a glass of orange drink, hot chocolate ${ }^{2}$, coffee or red wine ${ }^{3}$ too. Orange drink and oatmeal contain lots of vitamin D. Small quantities of supplements showed improvement of our body functions. But remember the best sources of nutrients come from natural sources. After a gentle breakfast a college student usually takes an Omega-3 capsule such as cod liver oil capsules - there are three main omega-3s: EPA, DHA and ALA. EPA and DHA are the primary omega-3s you need to support your heart and your mind and can be found in fatty fish and omega-3 supplements.[4] ALA (alpha-linolenic acid) is a true "essential" omega-3 because our bodies can't make it on its own. We need to get ALA from our diet by consuming ALA-rich foods like flax seeds. ALA is a precursor to EPA and DHA, but the conversion rate in our bodies is extremely low. In fact, 50-60\% of the solid matter found in the brain is fat, and the quality of the fat is what is most important. Unlike the fat that is deposited and packed in our thighs and abdomen, brain fat is a major structural component that plays a vital role in how our brain functions. - Additionally, a student may take ginkgo biloba capsules after the breakfast and after the lunch too. Study showed that in regard with cognition enhancement, taking a ginkgo biloba capsule plus a balanced nootropic capsule, containing essential vitamins, minerals, and phosphatidylserine (e.g. Neurozan) has at least a same effect as an artificial (abusive) stimulant drug stimulants, temporarily increase alertness and energy - such as Ritalin and amphetamines, meanwhile it (a premium nootropic supplement) has some benefits for your body. A magnesium tablet a day can boost your mental energy levels, you may take a tablet after a meal in the morning or noon. Recent studies proved consumption of vitamin B12 improves cognitive functions, and even makes new neural-connections inside the brain, however it may cause unwanted adverse effects. Vitamin B12 deficiency can cause almost any psychiatric symptom-from anxiety, and panic to depression and hallucinations. GABA is a naturally occurring neurotransmitter in our brain, which as one of your body's primary neurotransmitters is crucial to calm down the central nervous system (CNS). Neurotransmitters are chemicals which enable the brain cells to transmit impulses from one to another. Making a balanced level of GABA inside the brain usually aids relaxation, sleep, and also concentration and focus, in attention-deficit syndromes. Since it's difficult for a GABA supplement to cross the blood brain barrier (BBB) - the blood-brain barrier is a highly selective semipermeable membrane barrier that separates the circulating blood from the brain and extracellular fluid in the CNS - benzodiazepine drugs regulate this neurotransmitter functions inside the brain. Certainly, college students of the 90 s and 2000s were not the first group to get hooked on prescription stimulants. Coffee is another natural

\footnotetext{
${ }^{2}$ Today, chocolate is lauded for its tremendous antioxidant potential. The higher the cocoa content, the more health benefits there are and the less sugar content, which is better for overall health.

${ }^{3}$ Antioxidants in red wine called polyphenols may help protect the lining of blood vessels in your heart. A polyphenol called resveratrol is one substance in red wine that's gotten attention.
} 
stimulant that over moderate consumption would give you some benefits. It improves the average mental functions for its stimulant effects and since it can increase the level of GABA up to $65 \%$, these high amounts of GABA inside our brain may lead to increased anxiety. Consumption of vitamin $\mathrm{C}$ plus Coenzyme Q10 plus Zinc tablets not only slows down your brain's cells aging process but noticeably could make your skin healthy and beautiful. Curcumin capsules - made of turmeric essence (Curcuma longa L.) - contains another form of antioxidants. Eating parsley with breakfast and kiwis and red grapes with lunch would improve the aging process amazingly. Phosphatidylserine is a powerful cognitive enhancer, but if it's hard for you to find this supplement you can boost your body's level of phosphatidylserine by eating a piece of Atlantic mackerel, Atlantic herring, or half a chicken heart. These meats contain lots of fat so you should not serve a lot. Mostly, phosphatidylserine foods are high-fat foods, including HDL cholesterol (good cholesterol), and also some LDL cholesterol (bad cholesterol), usually extracted from bovine, soybeans, chicken hearts, etc. Chicken meat, itself, is a healthy meat for everybody. It contains good proteins. Don't get a drug supplement over dose, and you shall not take the supplementations over long term periods of time and also when you are on vacation. Three months or longer means a long period of time. Don't take a multivitamin capsule except that your doctor prescribed it to be taken for a short period of time (1-2 months), since man-made multivitamins sometimes caused abnormal cell growth in women. Either feeding body cells with premium fuel is an important factor, the other issues raise as some people like to eat raw meat, which is not frozen well. Sometimes it caused fatal diseases by parasites. Olive oil, tomatoes, and ginger roots are natural anti-inflammatory foods that are required to be consumed with antioxidants. Seriously limit pizza and red meat in your diet plan.[3] But don't forget fish and chicken meat in your diet since these foods are premium sources of proteins and essential amino-acids. Don't forget to exercise regularly as it burns your body fat and relive your body from oxidative stress. Eat the bites of bread well. Thus, you dramatically decrease the rate of getting cancers or getting old. So, reduce the anxiety, reduce your anxiety as you are somewhere made the Eucharist. Incorrect exercises are destructive for body's anatomy. Touch the spirit of humanity, shall grow internal happiness for all, in the mid-stream of a sensible life of routines. The better you chew, the better you digest nutrition. Chewing breaks your food down from large particles into smaller particles that are more easily digested. The longer you chew, the more time it will take you to finish a meal. And research shows that eating slowly can help you to eat less and, ultimately, to avoid weight gain or even helps you to lose weight. An average woman needs to eat about 2000 calories per day. Navy beans, cheese, eggs, cranberries, organic strawberries, and sea vegetables contain iodine. Kale, spinach, broccoli, beans, and lentils not only are good sources of iron, antioxidants, and vitamin $\mathrm{K}$, but also these vegetables would help you during Premenstrual syndrome (PMS). Citrus fruits, peppers, tomatoes, carrots, sweet potatoes, and nuts contain powerful antioxidants too, they are rich sources of vitamin A. Corn contains certain B vitamins and 
vitamin $\mathrm{C}$, as well as magnesium and potassium. It is a good source of two antioxidants, zeaxanthin and lutein. These foods and fruits protect you from cancer. Serve a tasty dish of Brunswick stew if you feel tired during PMS.

Eggs, dates, lentils, and bananas considered as regular and somehow athletic foods, containing high energy level. But not only these foods work well for your body muscles if you go to gym but also for your digestive system and the brain, but remember it could be better that you don't eat such foods too much every day, not to get obesity, etc. If you serve many foods containing high amounts of nutrition so just consume the necessary and required supplements and vice versa. By maintaining a dietary balance between foods and supplements not only you would enhance your brain's cognitive functions, but also you may live a much longer and healthier life. You may switch between a habitual form of antioxidant supplement during short periods of drug consumption, each time. And keep eating greens and highly nutritious foods.

\section{References}

[1] Kara Rogers (ed.). Sir frederick gowland hopkins and his "accessory food factors". Britannica, Revised 2007.

[2] Stephan Meier-Oeser; Edward N. Zalta (ed.). Medieval semiotics. The Stanford Encyclopedia of Philosophy, Summer 2011 Edition.

[3] Natalie Butler; Kimberly Holland. Eating with high blood pressure: Food and drinks to avoid. Healthline, 2016.

[4] Ph.D. Jane Higdon. Essential fatty acids. Linus Pauling Institute Oregon State University, 2003.

[5] David Klahr. Information processing approaches to cognitive development. Carnegie Mellon University, July 1, 1988.

[6] Jessica Martinez. Mediterranean and asian diets. livestrong.com, Oct 03, 2017.

[7] Saul McLeod. Cognitive psychology. simplypsychology.org, 2007.

[8] George A. Miller. The cognitive revolution: a historical perspective. Department of Psychology, Princeton University, 2003.

[9] Kim Ogonosky. Eatfitters. adventuresinanewishcity.com, October 9, 2015.

[10] Lilian Cheung; Brett Otis; Nancy Oliveira. Antioxidants: Beyond the hype. Harvard T.H. Chan - School of Public Health, (2017, Retrieved).

[11] Jie Wang; Jing Yi. Cancer cell killing via ros: To increase or decrease, that is the question. Taylor $\mathscr{E}$ Francis, Dec 01, 2008. 